\title{
Lusternik-Schnirelmann category, complements of skeleta and a theorem of Dranishnikov
}

\author{
JOHN OPREA \\ JEFF STROM
}

\begin{abstract}
In this paper, we study the growth with respect to dimension of quite general homotopy invariants $\mathcal{Q}$ applied to the $\mathrm{CW}$ skeleta of spaces. This leads to upper estimates analogous to the classical "dimension divided by connectivity" bound for LusternikSchnirelmann category. Our estimates apply, in particular, to the Clapp-Puppe theory of $\mathcal{A}$-category. We use $\operatorname{cat}^{1}(X)$ (which is $\mathcal{A}$-category with $\mathcal{A}$ the collection of 1-dimensional CW complexes), to reinterpret in homotopy-theoretical terms some recent work of Dranishnikov on the Lusternik-Schnirelmann category of spaces with fundamental groups of finite cohomological dimension. Our main result is the inequality $\operatorname{cat}(X) \leq \operatorname{dim}\left(B \pi_{1}(X)\right)+\operatorname{cat}^{1}(X)$, which implies and strengthens the main theorem of Dranishnikov [7].
\end{abstract}

Errata Minor corrections made 11 August 2010

55M30; 55P99

\section{Introduction}

The Lusternik-Schnirelmann category of a space $X$, denoted cat $(X)$, is the smallest integer $k$ so that $X$ can be covered by open sets $U_{0}, U_{1}, \ldots, U_{k}$, each of which is contractible to a point in $X$. LS category is an important numerical invariant in algebraic topology, critical point theory and symplectic geometry (see, for instance, Cornea et al [5], Clapp and Puppe [4] and Rudyak and Oprea [22]). One of the most basic estimates for LS category is (by Grossman [12] or see, for instance, Cornea et al [5])

$$
\operatorname{cat}(X) \leq \frac{\operatorname{dim}(X)}{c+1},
$$

where $X$ is $c$-connected. While this inequality was originally proven for covering dimension, the fact (shown later) that LS category is a homotopy invariant allows us to take $\operatorname{dim}(X)$ to mean homotopy dimension, the minimum covering dimension of all spaces homotopy equivalent to $X$. For $\mathrm{CW}$ or simplicial complexes, covering 
dimension is the same as cellular or simplicial dimension, so for these spaces the term homotopy dimension means the minimum cellular or simplicial dimension of all spaces homotopy equivalent to $X$. Throughout the paper, "dim" shall mean homotopy dimension whenever we speak of it in the context of a homotopy invariant.

Note that the inequality (\$) implies that a space whose category is greater than half the dimension must have a nontrivial fundamental group. In the world of closed 3-manifolds, the fundamental group completely determines the category (see GómezLarrañaga and González-Acuña [10], Oprea and Rudyak [19] or Švarc [26, Corollary (attributed to Berstein), page 117]). But such a result is too much to hope for in general, and the precise influence of the fundamental group on the category of the space is still rather a mystery.

In [3] (also see Švarc [26] and Dranishnikov and Rudyak [9]), Berstein discovered a very interesting connection between the fundamental group and category. If cat $(X)=$ $\operatorname{dim}(X)=n$, then there must be a class $\alpha \in H^{1}(X ; I(\pi))$ with $\alpha^{n} \neq 0$. Here, $I(\pi)$ denotes the augmentation ideal of the group ring on the fundamental group $\pi=\pi_{1}(X)$ and cohomology is taken with local coefficients in $I(\pi)$. The same local coefficient approach has been used in [8] to show that manifolds with category equal to 2 are either surfaces or have free fundamental groups. This has given rise to further study of the LS category of CW complexes with free fundamental group (see Dranishnikov, Katz and Rudyak [8], J Strom [25] and Dranishnikov [6]).

These results led Rudyak to conjecture that the LS category of spaces whose fundamental groups have finite cohomological dimension should be asymptotically bounded above by $\frac{1}{2} \operatorname{dim}(X)$. Rudyak's conjecture was reformulated by Dranishnikov as the explicit upper bound

$$
\operatorname{cat}(X) \leq \operatorname{dim}\left(B \pi_{1}(X)\right)+\left\lceil\frac{\operatorname{dim}(X)-1}{2}\right\rceil .
$$

In the papers [6; 7], Dranishnikov proved this conjecture, first for groups with cohomological dimension $\leq 2$ and then for arbitrary groups. The inequality is sharp for $X=S^{1}$, but other examples seem difficult to come by. Examples such as $X=B \pi \times \mathbb{C P}^{n}$ suggest that it might be possible to reduce the upper bound.

Problem 1.1 Is it true that

$$
\operatorname{cat}(X) \leq\left\lceil\frac{\operatorname{dim}\left(B \pi_{1}(X)\right)+\operatorname{dim}(X)-1}{2}\right\rceil ?
$$

We do not address this problem here, although an implication is mentioned in Section 7.

For his proof of the conjecture, Dranishnikov introduced some novel and useful techniques for the estimation of LS category. The approach is point-set theoretic, 
involving clever manipulation of open covers, and so it does not fit neatly into the homotopy-theoretical study of LS category.

This paper grew out of our attempts to understand and reinterpret Dranishnikov's argument from a homotopy-theoretic point of view. We found that the proof of the main lemma [7, Lemma 3.2] implies a bit more than the lemma states: namely, there is an inequality between $\operatorname{cat}(X)$ and a certain invariant of $X$, which we provisionally call $\Gamma_{1}(X)$ (it is unnamed in Dranishnikov's paper). We prove here that $\Gamma_{1}(X)$ is in fact the 1 -category $\operatorname{cat}^{1}(X)$ defined by Clapp and Puppe [4]. Thus we have the following formula.

\section{Theorem 1.2 (Corollary 6.2)}

$$
\operatorname{cat}(X) \leq \operatorname{dim}\left(B \pi_{1}(X)\right)+\operatorname{cat}^{1}(X) \leq \operatorname{dim}\left(B \pi_{1}(X)\right)+\left\lceil\frac{\operatorname{dim}(X)-1}{2}\right\rceil .
$$

A great deal of the complexity of Dranishnikov's proof arises in order to estimate $\Gamma_{1}(X)$. Once we have shown that this is simply $\operatorname{cat}^{1}(X)$, we can find this upper estimate by a different and more homotopy-theoretically satisfying method. Our alternative method depends on a fundamental relation between the skeleta of a simplicial complex and their complements (see Lemma 2.2), which implies the following estimate of the homotopy dimension of the complement of a skeleton.

Proposition 1.3 If $X$ is an $n$-dimensional simplicial complex and $X_{r}$ is the $r-$ skeleton of $X$, then $X_{n}-X_{r}$ has the homotopy type of a complex of dimension at most $n-r-1$.

This must certainly be known to geometric topologists, but we have not been able to find it in the literature. Moreover, we have found the result to be an extremely useful tool that is a common thread for results throughout the paper, so we give a proof in Section 2.

When $X$ is a simplicial complex, Lemma 2.2 implies a result of $\operatorname{Roth}$ : $\operatorname{cat}\left(X_{n}-X_{r}\right) \leq$ $n-r-1$ if $X_{n}-X_{r}$ is path-connected [21, Lemma 1.6]. We must mention, though, that Roth's lemma applies to general CW complexes, while ours is valid only for simplicial complexes. Roth used his lemma - in its full generality - to derive an upper bound on the LS category of unordered configuration spaces.

More importantly for us however, Lemma 2.2 is the key to a completely new proof of the fundamental superadditivity property of categorical sequences (see Nendorf, Scoville and Strom [17]). This new proof is easily generalized, and reveals that superadditivity is actually a feature of the sequences defined in an analogous way for 
a vast array of homotopy invariants $\mathcal{Q}$. Superadditivity of the $\mathcal{Q}$-sequence implies that $\mathcal{Q}(X)$ is bounded above by $\left\lfloor\operatorname{dim}(X) /\left(\operatorname{conn}^{\mathcal{Q}}(X)+1\right)\right\rfloor$, where conn ${ }^{\mathcal{Q}}(X)$ is the $\mathcal{Q}$-connectivity of $X$, defined below in Section 3. This theory applies, in particular, to 1 -category [4], yielding the bound $\operatorname{cat}^{1}(X) \leq\lceil(\operatorname{dim}(X)-1) / 2\rceil .{ }^{1}$

The conjecture proved by Dranishnikov (and reproved here) evolved from a conjecture in an earlier draft of the paper [8], which asked whether compact manifolds of dimension $\geq 4$ and with free fundamental group must satisfy cat $(M)<\operatorname{dim}(M)-1$. In [25], the second author showed that in fact for any CW complex $X$ with free fundamental group, $\operatorname{cat}(X)$ is bounded above by, roughly, $\frac{2}{3} \operatorname{dim}(X)$, which was good enough to prove the conjecture above for all manifolds with $\operatorname{dim}(M)>4$. The case $\operatorname{dim}(M)=4$ remained maddeningly out of reach, even for the much stronger result of Dranishnikov [6].

However, Brandon Strom [24] has called our attention to a lemma of Hillman [13]: a 4-dimensional Poincaré duality complex with free fundamental group has the homotopy type of a CW complex built by attaching a single 4-cell to a wedge of lower dimensional spheres. Thus all such spaces have category at most 2, which resolves, in the affirmative, the $n=4$ case of the original question. (In fact, while Hillman's result applies to Poincaré duality complexes, Matumoto and Katanaga [16] had earlier proved the same result for 4-dimensional manifolds and both these results were mentioned in the published version of $[8] .^{2}$ )

These results also answer an old question about symplectic manifolds. Since every finitely presented group arises as the fundamental group of a closed symplectic 4-manifold, we now know that there exist non-simply connected symplectic manifolds $M^{4}$ (with free fundamental group) whose category is half their dimension. No examples of such spaces were previously known (although a cohomologically symplectic example was constructed in [2]). By Poincaré duality, a 4-manifold with a nontrivial free fundamental group must have a nontrivial cup product in its cohomology, which means that

$$
\operatorname{cat}\left(M^{4} \times \prod_{1}^{s} S^{2}\right)=s+2=\frac{\operatorname{dim}\left(M^{4} \times \prod_{1}^{s} S^{2}\right)}{2},
$$

and so such symplectic manifolds exist in all even dimensions. In Section 7, we shall discuss some further applications of our results to symplectic topology.

\footnotetext{
${ }^{1}$ We use Dranishnikov's bound here, but note that for an integer $r>1$ and any integer $n,\lfloor n / r\rfloor \leq$ $\lceil(n-1) / r\rceil$ with equality holding when $r=2$.

${ }^{2}$ Thanks to the referee for pointing these items out.
} 
Acknowledgement We thank the Midwest Topology Network for funding that allowed the second author to visit the first in September and October 2009, when the results of this paper were conceived.

\section{The fundamental dimension lemma}

If we remove the $k$-skeleton from an $n$-dimensional simplicial complex, the result still has topological dimension $n$, but what is its homotopy dimension? A subsimplex $\sigma \subseteq \tau$ of the simplex $\tau$ has a complementary simplex $\check{\sigma}$ whose vertices are the vertices of $\tau$ that do not belong to $\sigma$. It follows that $\tau$ is equal to the join $\sigma * \check{\sigma}$ and that $\operatorname{dim}(\sigma)+\operatorname{dim}(\check{\sigma})=\operatorname{dim}(\tau)-1$. Thus the $n$-dimensional simplex $\tau$ is the standard homotopy pushout of the diagram $\sigma \leftarrow \sigma \times \check{\sigma} \rightarrow \check{\sigma}$, where $\operatorname{dim}(\sigma)=\ell$ and $\operatorname{dim}(\check{\sigma})=n-\ell-1$. This simple observation is the underlying idea in our result about the complements of skeleta.

We begin with a bit of groundwork. For basic results about simplicial complexes, see Seifert and Threlfall [23] and Alexandrov [1] for example.

Lemma 2.1 Let $\tau$ be an $n$-dimensional simplex, and let $\sigma$ be a $k$-dimensional simplex of the barycentric subdivision $\operatorname{sd}(\delta)$ of $\delta$. Then $\sigma \cap \operatorname{sd}\left(\Delta_{r}^{n}\right)$ is a single simplex $\sigma_{r}$ of dimension $\ell$ such that $r-(n-k) \leq \ell \leq r$.

Proof If $r=n$, the statement is plainly true; in particular, it holds for the barycentric subdivision of a 0 -simplex. We now assume that $0 \leq r<n$, and that the result is known for any $(n-1)$-simplex.

Let $\delta$ be an $n$-simplex, and let $\sigma$ be a $k$-simplex in $\operatorname{sd}(\delta)$. The $r$-skeleton $\delta_{r}$ is contained in the union of the $(n-1)$-faces of $\delta$. Therefore, if $\sigma$ does not include the barycenter of $\delta$ among its vertices, then $\sigma$ lies in an $(n-1)$-dimensional face $\tau$ of $\delta$ and, by the inductive hypothesis, $\sigma \cap \operatorname{sd}\left(\delta_{r}\right)=\sigma \cap \operatorname{sd}\left(\tau_{r}\right)$ is a single simplex $\sigma$ with dimension $\ell$ where

$$
r-(n-k)<r-((n-1)-k) \leq \ell \leq r .
$$

If, on the other hand, $\sigma$ does include the barycenter of $\delta$ among its vertices, then consider the $(k-1)$-dimensional simplex $\tau \subseteq \sigma$ complementary to the barycenter of $\delta$. We have $\sigma \cap \operatorname{sd}\left(\delta_{r}\right)=\tau \cap \operatorname{sd}\left(\delta_{r}\right)$ and the first part of the argument, applied to $\tau \subseteq \delta_{n-1}$, shows that $\tau \cap \operatorname{sd}\left(\delta_{r}\right)$ is a simplex of dimension $\ell$ with

$$
r-(n-k)=r-((n-1)-(k-1)) \leq \ell \leq r .
$$

Note that the condition $r-(n-k) \leq \ell$ is equivalent to $k-\ell-1 \leq n-r-1$. 
Lemma 2.2 (Fundamental Dimension Lemma) If $X$ is a simplicial complex, then its $n$-skeleton $X_{n}$ is homeomorphic to the standard homotopy pushout ${ }^{3}$ in the diagram

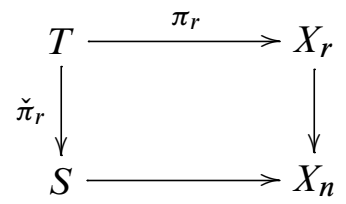

where

- $X_{r}$ is the $r$-skeleton of $X$,

- $\operatorname{dim}(T)<n$,

- $S \simeq X_{n}-X_{r}$ and $\operatorname{dim}(S)<n-r$.

Proof The idea of the proof is to show that it is true for a single simplex $\sigma$ of $\operatorname{sd}(X)$, in such a way that the maps and homotopies involved are compatible for the faces of the simplex. Then we piece them together to get the result for any assembly of simplices.

Let $\sigma$ be a $k$-simplex of the first barycentric subdivision $\operatorname{sd}\left(\Delta^{n}\right)$. By Lemma 2.1, the intersection $\sigma \cap \operatorname{sd}\left(\Delta_{r}^{n}\right)$ is a single simplex $\sigma(r)$ of dimension $\ell$ with $\ell \leq r$ and $k-\ell-1 \leq n-r-1$. Write $\check{\sigma}(r)$ for the simplex in $\sigma$ complementary to $\sigma(r)$, so that $\sigma$ is the join $\sigma(r) * \check{\sigma}(r)$, and $m=\operatorname{dim}(\check{\sigma}(r))=k-\ell-1 \leq n-r-1$.

Using the join notation in which points of the join are affine combinations $a t+b(1-t)$ with $t \in[0,1]$, we write

$$
\begin{aligned}
& \overline{\sigma(r)}=\left\{a t+b(1-t) \mid a \in \sigma(r), b \in \check{\sigma}(r) \text { and } t \geq \frac{1}{2}\right\}, \\
& \overline{\sigma(r)}=\left\{a t+b(1-t) \mid a \in \sigma(r), b \in \check{\sigma}(r) \text { and } t \leq \frac{1}{2}\right\} .
\end{aligned}
$$

Then we define

$$
\tau=\overline{\sigma(r)} \cap \overline{\check{\sigma}(r)}=\left\{\frac{1}{2}(a+b) \mid a \in \sigma(r), b \in \check{\sigma}(r)\right\},
$$

which is homeomorphic to $\sigma(r) \times \check{\sigma}(r)$ in such a way that the projection maps $p_{\sigma(r)}: \tau \rightarrow \sigma(r)$ and $p_{\check{\sigma}(r)}: \tau \rightarrow \check{\sigma}(r)$ are given by the formulas

$$
p_{\sigma(r)}\left(\frac{1}{2}(a+b)\right)=a \quad \text { and } \quad p_{\check{\sigma}(r)}\left(\frac{1}{2}(a+b)\right)=b .
$$

It is crucial to note that if $\sigma$ is contained in a larger simplex $\widetilde{\sigma}$, and we apply our construction to $\widetilde{\sigma}$, then its restriction to $\sigma$ is the same as the construction applied directly to $\sigma$. This is what makes it possible to glue our homotopy pushout diagrams together.

\footnotetext{
${ }^{3}$ ie, double mapping cylinder
} 
Clearly, $\overline{\sigma(r)}$ and $\overline{\sigma(r)}$ are (homeomorphic to) the mapping cylinders of $p_{\sigma(r)}$ and $p_{\check{\sigma}(r)}$. Since their intersection is precisely $\tau, \sigma$ is the (standard) homotopy pushout of the diagram

$$
\check{\sigma}(r) \stackrel{p_{\sigma}(r)}{\longleftarrow} \tau \stackrel{p_{\sigma(r)}}{\longrightarrow} \sigma(r) .
$$

Now for the general case: let $X$ be a simplicial complex, and define

$$
T=\bigcup_{\sigma \in \operatorname{sd}\left(X_{n}\right)} \overline{\sigma(r)} \cap \overline{\check{\sigma}(r)} \text { and } S=\bigcup_{\sigma \in \operatorname{sd}\left(X_{n}\right)} \check{\sigma}(r) .
$$

Note that $\operatorname{dim}(S) \leq n-r-1$. The union $\operatorname{sd}\left(X_{r}\right)=\bigcup_{\sigma \in \operatorname{sd}\left(X_{n}\right)} \sigma(r)$, while vitally important, does not need new notation. The maps $p_{\sigma(r)}$ and $p_{\check{\sigma}(r)}$ for the various simplices $\sigma$ of $\operatorname{sd}(X)$ fit together to define maps $\pi_{r}: T \rightarrow X_{r}$ and $\check{\pi}_{r}: T \rightarrow S$. Explicitly,

$$
\pi_{r}(x)=p_{\sigma(r)}(x) \text { and } \check{\pi}_{r}(x)=p_{\check{\sigma}(r)}(x),
$$

where $x \in \sigma$, which are well defined because of the observation in the previous paragraph. Then we define

$$
\overline{X_{r}}=\bigcup_{\sigma \in \operatorname{sd}\left(X_{n}\right)} \overline{\sigma(r)} \text { and } \bar{S}=\bigcup_{\sigma \in \operatorname{sd}\left(X_{n}\right)} \overline{\sigma(r)},
$$

which are the mapping cylinders of $\pi_{r}$ and $\check{\pi}_{r}$, respectively. Their intersection is equal to $T$ and their union is $X_{n}$, so $X_{n}$ is homeomorphic to the double mapping cylinder of the diagram

$$
X_{r} \stackrel{\pi_{r}}{\longleftarrow} \stackrel{\check{\pi}_{r}}{\longrightarrow} S .
$$

The proof is complete since $S \simeq \bar{S} \simeq X_{n}-X_{r}$ where, as mentioned before, $\operatorname{dim}(S) \leq$ $n-r-1$.

\section{Growth of homotopy invariants with dimension}

The categorical sequence of the space $X$ is the function $\sigma_{X}: \mathbb{N} \rightarrow \mathbb{N} \cup\{\infty\}$ defined by

$$
\sigma_{X}(k)=\inf \left\{n \mid \operatorname{cat}\left(X_{n} \rightarrow X\right) \geq k\right\},
$$

where $X_{n} \rightarrow X$ denotes an $n$-skeleton of $X{ }^{4}$ The sequence $\sigma_{X}$ describes how the Lusternik-Schnirelmann category grows with respect to the dimension of the CW skeleta of $X$. Categorical sequences were introduced and applied in [17; 25]. Some key properties of categorical sequences $\sigma_{X}$ are: $\sigma_{X}$ is independent of the choice of

\footnotetext{
${ }^{4}$ This is terminology from [17]: an $n$-skeleton for a general space $X$ is an $n$-equivalence $X_{n} \rightarrow X$ in which $X_{n}$ is a CW complex with $\operatorname{dim}\left(X_{n}\right) \leq n$.
} 
CW decomposition of $X$; the finite values of $\sigma_{X}$ are strictly increasing; and that the sequence is superadditive.

In this section we show that a profitable theory of sequences can be developed for any invariant $\mathcal{Q}$ of homotopy classes of maps satisfying the following three properties: ${ }^{5}$

(1) (Normalization) $\mathcal{Q}(*)=0$.

(2) (Factorization) In a homotopy commutative triangle

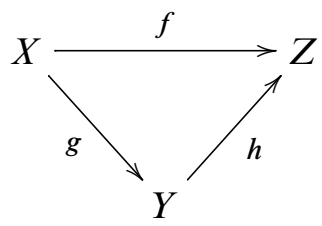

$\mathcal{Q}(f) \leq \min \{\mathcal{Q}(g), \mathcal{Q}(h)\}$.

(3) (Pushout) In a homotopy commutative diagram

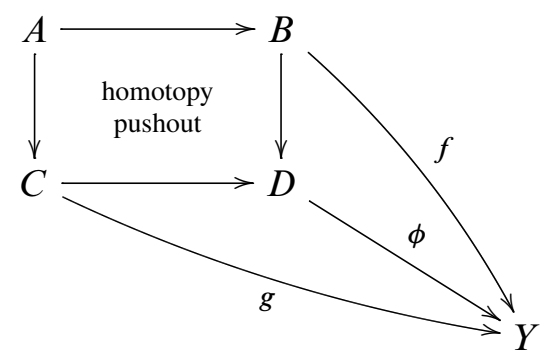

$$
\mathcal{Q}(\phi) \leq \mathcal{Q}(f)+\mathcal{Q}(g)+1
$$

For such $\mathcal{Q}$ we define, for any space $X$, the $\mathcal{Q}$-sequence $\sigma_{X}^{\mathcal{Q}}: \mathbb{N} \rightarrow \mathbb{N} \cup\{\infty\}$ by setting

$$
\sigma_{X}^{\mathcal{Q}}(k)=\inf \left\{n \mid \mathcal{Q}\left(X_{n} \rightarrow X\right) \geq k\right\}
$$

where, as before, $X_{n} \rightarrow X$ is an $n$-skeleton. A numerical invariant $\mathcal{Q}$ of homotopy classes of maps gives rise to an invariant of spaces by the simple rule

$$
\mathcal{Q}(X)=\mathcal{Q}\left(\mathrm{id}_{X}\right),
$$

and the sequence $\sigma_{X}^{\mathcal{Q}}$ is a useful tool for computing $\mathcal{Q}(X)$, because

$$
\mathcal{Q}(X)=\max \left\{k \mid \sigma_{X}^{\mathcal{Q}}(k)<\infty\right\},
$$

provided $X$ is a finite-dimensional CW complex.

${ }^{5}$ Of course, we are thinking of an invariant $\mathcal{Q}$ such as the category of a map when we define these properties, but there are more general invariants that satisfy them, as we shall see in Section 4. 
Remark 3.1 The Factorization Property implies that

(1) if $f: X \rightarrow Y$, then $\mathcal{Q}(f) \leq \min \{\mathcal{Q}(X), \mathcal{Q}(Y)\}$, and

(2) if $X$ is a retract of $Y$, then $\mathcal{Q}(X) \leq \mathcal{Q}(Y)$.

The three conditions imply that the sequence $\sigma_{X}^{\mathcal{Q}}$ is well-behaved in a variety of ways.

Proposition 3.2 Suppose $\mathcal{Q}$ satisfies the Normalization, Factorization and Pushout Properties.

(1) The sequence $\sigma_{X}^{\mathcal{Q}}$ is independent of the choice of CW approximation: it is an invariant of the weak homotopy type of $X$.

(2) If $X$ is path-connected, then the finite values of $\sigma_{X}^{\mathcal{Q}}$ are strictly increasing.

(3) If $X$ is simply connected and $\sigma_{X}^{\mathcal{Q}}(k)=n$, then $H^{n}(X ; G) \neq 0$ for some coefficients $G$.

Proof Part (1) follows from Cellular Approximation. If $i: X_{n} \rightarrow X$ is an $n$-skeleton and $j: \bar{X}_{m} \rightarrow X$ is an $m$-skeleton with $n \leq m$ (not necessarily from the same CW decomposition), then we have a homotopy commutative diagram

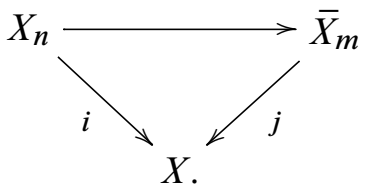

Using the Factorization Property, this shows $\mathcal{Q}(i) \leq \mathcal{Q}(j)$ in general, and when $m=n$, it implies $\mathcal{Q}(i)=\mathcal{Q}(j)$.

To prove (2), choose a CW approximation for $X$ so that there are cofiber sequences $\bigvee S^{n} \rightarrow X_{n} \rightarrow X_{n+1}$. The Pushout Property and the Normalization Property together give $\mathcal{Q}\left(X_{n+1}\right) \leq \mathcal{Q}\left(X_{n}\right)+1$. Because of (1) we may use these skeleta to compute $\sigma_{X}^{\mathcal{Q}}$. Now the weakly increasing sequence of numbers $\left\{\mathcal{Q}\left(X_{n}\right)\right\}$ has no gaps until it gets to $\infty$. Therefore if $\sigma_{X}^{\mathcal{Q}}(k)=n$, then $\mathcal{Q}\left(X_{n-1} \rightarrow X\right)=k-1$ (as opposed to $\left.\mathcal{Q}\left(X_{n-1} \rightarrow X\right) \leq k-1\right)$, and, finally, $\sigma_{X}^{\mathcal{Q}}(k-1)<\sigma_{X}^{\mathcal{Q}}(k)$.

For the last statement, note if $X$ is simply connected and $H^{n}(X ; G)=0$ for all coefficients $G$, then $X$ has a CW decomposition in which $X_{n}=X_{n-1}$, which contradicts $\sigma_{X}^{\mathcal{Q}}(k)=n$ (see Strom [25, Remark 3] for more detail).

Now we come to the big surprise: the Pushout Property implies that the $\mathcal{Q}$-sequence is superadditive. 
Theorem 3.3 Suppose $Q$ satisfies the Factorization and Pushout Properties. Then the sequence $\sigma_{X}^{\mathcal{Q}}$ is superadditive, in the sense that

$$
\sigma_{X}^{\mathcal{Q}}(k+l) \geq \sigma_{X}^{\mathcal{Q}}(k)+\sigma_{X}^{\mathcal{Q}}(l)
$$

for all $k, l \in \mathbb{N}$.

Proof Let $\sigma_{X}^{\mathcal{Q}}(k)=n$ and $\sigma_{X}^{\mathcal{Q}}(l)=m$. To show $\sigma_{X}^{\mathcal{Q}}(k+l) \geq n+m$, we consider particular skeleta

$$
i_{n+m-1}: X_{n+m-1} \rightarrow X, \quad i_{m-1}: X_{m-1} \rightarrow X \quad \text { and } \quad i_{n-1}: X_{n-1} \rightarrow X .
$$

Since $\mathcal{Q}$ satisfies the Factorization Property, we may use any skeleta we like. Since a CW complex has the homotopy type of a simplicial complex (see Lundell and Weingram [15]), we choose our $(n+m-1)$-skeleton $X_{n+m-1}$ to be a simplicial complex, and our $(n-1)$ - and $(m-1)$-skeleta be the simplicial skeleta of $X_{n+m-1}$. Since $\sigma_{X}^{\mathcal{Q}}(k)=n$ and $\sigma_{X}^{\mathcal{Q}}(l)=m$, we have $\mathcal{Q}\left(i_{n-1}\right)<k$ and $\mathcal{Q}\left(i_{m-1}\right)<l$; we will show that $\mathcal{Q}\left(i_{n+m-1}\right)<k+l$.

Our Fundamental Dimension Lemma gives the homotopy pushout square

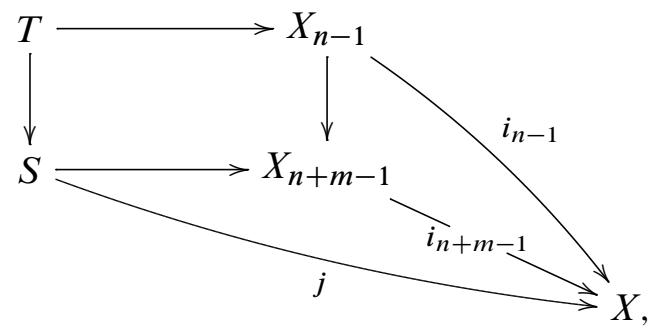

in which $\operatorname{dim}(S) \leq m-1$. Then the Pushout and Factorization Properties give

$$
\begin{aligned}
\mathcal{Q}\left(i_{n+m-1}\right) & \leq \mathcal{Q}\left(i_{n-1}\right)+\mathcal{Q}(j)+1 \\
& \leq \mathcal{Q}\left(i_{n-1}\right)+\mathcal{Q}\left(i_{m-1}\right)+1 \\
& \leq(k-1)+(l-1)+1 \\
& <k+l
\end{aligned}
$$

since, by Cellular Approximation, the inclusion $j: S \hookrightarrow X$ factors up to homotopy through $i_{m-1}: X_{m-1} \hookrightarrow X$.

Superadditivity provides us with a useful upper bound for $\mathcal{Q}(X)$ when $X$ is finitedimensional. To state it, we define the $\mathcal{Q}$-connectivity of $X$ to be the number

$$
\operatorname{conn}^{\mathcal{Q}}(X)=\sigma_{X}^{\mathcal{Q}}(1)-1 .
$$


As long as $\mathcal{Q}$ satisfies the Normalization Property, $\operatorname{conn}^{\mathcal{Q}}(X)$ is bounded below by the ordinary connectivity, conn $(X)$. If $\mathcal{Q}$ is ordinary Lusternik-Schnirelmann category, then $\operatorname{conn}^{\mathcal{Q}}(X)=\operatorname{conn}(X)$. We show that every numerical invariant satisfying the Normalization, Factorization and Pushout Properties has an upper bound of the form "dimension divided by connectivity."

Proposition 3.4 Suppose $\mathcal{Q}$ satisfies the Normalization, Factorization and Pushout Properties. If $X$ is $c$-connected, then

$$
\mathcal{Q}(X) \leq\left\lfloor\frac{\operatorname{dim}(X)}{\sigma_{X}^{\mathcal{Q}}(1)}\right\rfloor=\left\lfloor\frac{\operatorname{dim}(X)}{\operatorname{conn}^{\mathcal{Q}}(X)+1}\right\rfloor \leq\left\lfloor\frac{\operatorname{dim}(X)}{c+1}\right\rfloor .
$$

Proof If $X$ is infinite-dimensional, there is nothing to prove, so we assume that $X$ is finite-dimensional. By superadditivity, we have $\sigma_{X}^{\mathcal{Q}}(k) \geq k \cdot \sigma_{X}^{\mathcal{Q}}(1)$ for all $k$. Therefore

$$
\begin{aligned}
\mathcal{Q}(X) & =\max \left\{k \mid \sigma_{X}^{\mathcal{Q}}(k)<\infty\right\} \\
& =\max \left\{k \mid \sigma_{X}^{\mathcal{Q}}(k) \leq \operatorname{dim}(X)\right\} \\
& \leq \max \left\{k \mid k \cdot \sigma_{X}^{\mathcal{Q}}(1) \leq \operatorname{dim}(X)\right\} \\
& =\left\lfloor\frac{\operatorname{dim}(X)}{\sigma_{X}^{\mathcal{Q}}(1)}\right\rfloor .
\end{aligned}
$$

Remark 3.5 The Pushout Property is a homotopy-theoretical form of the covering definition of LS category. The covering definition has been used to prove that $\operatorname{cat}(X) \leq$ $\operatorname{dim}(X)$ (see Cornea et al [5, Theorem 1.7]), but we have not seen the covering definition yield the better bound $\operatorname{cat}(X) \leq \operatorname{dim}(X) /(\operatorname{conn}(X)+1)$. Furthermore, the introduction of $\mathcal{Q}$-connectivity is new, and leads to lower upper bounds.

\section{$4 \mathcal{A}$-Category}

Following Clapp and Puppe [4], ${ }^{6}$ we define the $\mathcal{A}$-category of a map $f: Z \rightarrow X$. An open cover $\mathcal{U}=\left\{U_{0}, U_{1}, \ldots, U_{k}\right\}$ of $Z$ is an $\mathcal{A}-L S$ cover for $f$ if each restriction $\left.f\right|_{U_{i}}$ factors, up to homotopy, through a space $A \in \mathcal{A}$; for such a cover, we set $c(\mathcal{U})=k$. Then we define

$$
\operatorname{cat}_{\mathcal{A}}(f)=\inf \{c(\mathcal{U}) \mid \mathcal{U} \text { is an } \mathcal{A} \text {-LS cover of } f\} .
$$

The theory we developed in the previous section applies to the invariants $\mathcal{Q}=$ cat $_{\mathcal{A}}$.

\footnotetext{
${ }^{6}$ Note that we use reduced category, where $\operatorname{cat}_{\mathcal{A}}(*)=0$ while Clapp and Puppe use unreduced category, where $\operatorname{cat}_{\mathcal{A}}(*)=1$.
} 
Theorem 4.1 (Clapp-Puppe [4, (1.3)]) For any $\mathcal{A}$, the invariant cat $\mathcal{A}_{\mathcal{A}}$ satisfies the Normalization, Factorization and Pushout Properties.

It follows that there are well defined, strictly increasing and superadditive sequences $\sigma_{X}^{\text {cat }} \mathcal{A}$; we'll use the simpler notation $\sigma_{X}^{\mathcal{A}}$ for these sequences. Theorem 4.1 implies that each invariant cat ${ }_{\mathcal{A}}$ has an upper bound of the form "dimension divided by connectivity." A related, but more technical, result was proved in [4, Lemma 5.7]. Let's write $\operatorname{conn}^{\mathcal{A}}$ for connectivity with respect to cat $\mathcal{A}_{\mathcal{A}}$.

Corollary 4.2 For a finite-dimensional $C W$ complex $X$,

$$
\operatorname{cat}_{\mathcal{A}}(X) \leq\left\lfloor\frac{\operatorname{dim}(X)}{\operatorname{conn}^{\mathcal{A}}(X)+1}\right\rfloor .
$$

Clapp and Puppe single out the case $\mathcal{A}_{r}=\{r$-dimensional spaces $\}$ as worthy of special attention and they suggest the notation cat ${ }^{r}$ for this special notion of category. For path-connected spaces, cat ${ }^{0}$ is ordinary LS category. Also, if $s \leq r$, then $\mathcal{A}_{s} \subseteq \mathcal{A}_{r}$ and so cat $^{r}(X) \leq \operatorname{cat}^{s}(X)$. In particular, $\operatorname{cat}^{r}(X) \leq \operatorname{cat}(X)$ for any space $X$. To obtain a tighter upper bound for $\operatorname{cat}^{r}(X)$, we need to estimate the connectivity $\operatorname{conn}^{r}(X)=$ $\operatorname{conn}^{\mathcal{A}}(X)$. The following result follows from the definitions and Proposition 3.2.

Lemma 4.3 (1) For any space $X$,

$$
r \leq \operatorname{conn}^{r}(X) \leq \inf \left\{m \geq r \mid H^{m+1}(X ; G) \neq 0 \text { for some abelian } G\right\} .
$$

(2) If $X$ is simply connected then the second inequality of (1) is actually an equality. In particular, $\operatorname{conn}^{r}(X) \geq r$ for all $X$.

Now we can establish our estimates for $\operatorname{cat}^{r}(X)$.

Proposition 4.4 Let $X$ be a space with $X_{r}$ the $r$-skeleton of $X$. Suppose that $h^{*}$ is a multiplicative cohomology theory. If $K=\operatorname{ker}\left(h^{*}(X) \rightarrow h^{*}\left(X_{r}\right)\right)$, then

$$
\operatorname{nil}(K) \leq \operatorname{cat}^{r}(X) \leq\left\lfloor\frac{\operatorname{dim}(X)}{r+1}\right\rfloor .
$$

where nil $(K)$ is the length of the longest nontrivial product of degree nonzero elements in $K$.

Proof The first inequality follows from [4, Proposition 3.1] and the standard cuplength inequality argument for category (see [5, Proposition 1.5]) since $K$ is precisely the set of elements of $h^{*}(X)$ that vanish when pulled back to any space of dimension at most $r$. The second inequality follows from Proposition 3.4 and Lemma 4.3. 
It is a standard consequence of the homotopy lifting property (see [5, Corollary 1.45 and Remark 1.46]) that $\operatorname{cat}(\bar{X}) \leq \operatorname{cat}(X)$ for any covering $\bar{X} \rightarrow X$. With a little care, the argument can be made to work for cat ${ }^{r}$ as well.

Lemma 4.5 If $p: \bar{X} \rightarrow X$ is a covering, then $\operatorname{cat}^{r}(\bar{X}) \leq \operatorname{cat}^{r}(X)$.

Proof Let $\operatorname{cat}^{r}(X)=n$, and let $U_{0}, \ldots, U_{k}$ be an $\mathcal{A}_{r}$-categorical cover of $X$. By composing with the homotopy compressing $U_{k}$ into the $r$-skeleton $X_{r}$, we obtain a homotopy $H: p^{-1}\left(U_{k}\right) \times I \rightarrow X$ with $H_{1}\left(p^{-1}\left(U_{k}\right)\right) \subset X_{r}$. By the homotopy lifting property, we have a homotopy $G: p^{-1}\left(U_{k}\right) \times I \rightarrow \bar{X}$ with $G_{1}\left(p^{-1}\left(U_{k}\right)\right) \subset p^{-1}\left(X_{r}\right)$. But, since $p$ is a local homeomorphism, we can always arrange cell structures so that $p^{-1}\left(X_{r}\right)=\bar{X}_{r}$. Hence, $p^{-1}\left(U_{k}\right)$ compresses into $\bar{X}_{r}$ for each $k$ and we therefore have $\operatorname{cat}^{r}(\bar{X}) \leq \operatorname{cat}^{r}(X)$.

Unfortunately, $\operatorname{cat}^{r}(\bar{X}) \leq \operatorname{cat}^{r}(X)$ is usually a poor estimate. For instance, the universal cover $S^{n} \rightarrow \mathbb{R} \mathrm{P}^{n}$ has cat $\left(S^{n}\right)=1$ and $\operatorname{cat}\left(\mathbb{R P}^{n}\right)=n$. Using our next proposition, we can do much better. The key is that if we know a bit about the homotopy groups of $X$, then we can sometimes obtain more useful information.

Proposition 4.6 Let $p: \tilde{X} \rightarrow X$ denote the universal covering. If $\pi_{j}(X)=0$ for $1<j \leq r$, then

$$
\operatorname{cat}(\tilde{X})=\operatorname{cat}^{r}(\tilde{X}) \leq \operatorname{cat}^{r}(X) \leq\left\lfloor\frac{\operatorname{dim}(X)}{r+1}\right\rfloor
$$

Proof Let cat $^{r}(X)=n$ with $\mathcal{A}_{r}$-categorical cover $X=U_{0} \cup U_{1} \cup \cdots \cup U_{n}$. Just as in the proof of Lemma 4.5, the homotopy lifting property implies that each $p^{-1}\left(U_{k}\right) \hookrightarrow \tilde{X}$ factors, up to homotopy, through an $r$-skeleton $\tilde{X}_{r} \rightarrow \tilde{X}$. Since $\pi_{j}(X)=0$ for $1<j \leq r$, we have $\pi_{j}(\tilde{X})=0$ for $1 \leq j \leq r$, and so the $r$-skeleton $\tilde{X}_{r} \rightarrow \tilde{X}$ is nullhomotopic. Therefore we have an LS-cover $\tilde{X}=p^{-1}\left(U_{0}\right) \cup p^{-1}\left(U_{1}\right) \cdots \cup p^{-1}\left(U_{n}\right)$ of $\tilde{X}$ and

$$
\operatorname{cat}(\tilde{X})=\operatorname{cat}^{r}(\tilde{X}) \leq \operatorname{cat}^{r}(X) \leq\left\lfloor\frac{\operatorname{dim}(X)}{r+1}\right\rfloor,
$$

by Proposition 3.4.

Example 4.7 Since $S^{n}$ is not contractible, we know cat $\left(S^{n}\right)>0$. From the universal covering $S^{n} \rightarrow \mathbb{R P}^{n}$, we obtain

$$
\operatorname{cat}\left(S^{n}\right)=\operatorname{cat}^{n-1}\left(S^{n}\right) \leq \operatorname{cat}^{n-1}\left(\mathbb{R P}^{n}\right) \leq\left\lfloor\frac{\left.\operatorname{dim}(\mathbb{R P})^{n}\right)}{(n-1)+1}\right\rfloor=1,
$$

so we recover $\operatorname{cat}\left(S^{n}\right)=1$ from the estimate for $\operatorname{cat}^{n-1}\left(\mathbb{R P}^{n}\right)$. 
Remark 4.8 Of course, in the situation of Proposition 4.6, the inequality $\operatorname{cat}(\tilde{X}) \leq$ $\lfloor\operatorname{dim}(X) /(r+1)\rfloor$ follows from the estimate $(+)$ of the Introduction. It is the fact that cat $^{r}(X)$ fits between these bounds that is of interest because it offers an opportunity for obtaining a better upper bound for the category of the universal cover of a space in terms of the category of the space.

Finally, we point out that general invariants $\mathcal{Q}$ are related to $\mathcal{A}$-category for carefully chosen collections $\mathcal{A}$.

Remark 4.9 Suppose $\mathcal{Q}$ satisfies the Normalization, Factorization and Pushout Properties and set $\mathcal{A}=\{A \mid \mathcal{Q}(A)=0\}$. Then $\mathcal{Q}(X) \leq \operatorname{cat}_{\mathcal{A}}(X)$. The reverse inequality is not generally true, for we could define

$$
\mathcal{Q}(X)=\left\{\begin{array}{l}
0 \text { if } \operatorname{cat}_{\mathcal{A}}(X)=0, \\
1 \text { otherwise }
\end{array}\right.
$$

This $\mathcal{Q}$ satisfies the three properties, but it is clearly not equal to cat ${ }_{\mathcal{A}}$.

\section{An LS type invariant of fiber pairs}

A fiber pair is simply a map of fibrations, ie, a strictly commutative diagram

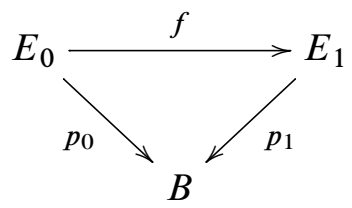

in which $p_{0}$ and $p_{1}$ are fibrations. If $F_{0}$ and $F_{1}$ are, respectively, the fibers of $p_{0}$ and $p_{1}$ over the basepoint $*$, then there is an induced map $F_{0} \rightarrow F_{1}$. If $B$ is path-connected, then the induced maps of fibers over other points of $B$ are homotopy equivalent to this map.

By forming the mapping cylinder over $B$, we may replace a fiber pair with an inclusion of a subfibration $E_{0} \hookrightarrow E_{1}$ over $B$. From now on, for conceptual (if not mathematical) convenience, we will work with inclusions of fibrations. An open cover $\mathcal{U}=\left\{U_{0}, U_{1}, \ldots, U_{n}\right\}$ of $E_{1}$ is a $\Gamma$-cover if each inclusion $U_{k} \hookrightarrow E_{1}$ factors through the inclusion $E_{0} \hookrightarrow E_{1}$, up to homotopy over $B$; for such a cover we define $\gamma(\mathcal{U})=n$. Then we set

$$
\Gamma\left(E_{0} \hookrightarrow E_{1}\right)=\inf \{\gamma(\mathcal{U}) \mid \mathcal{U} \text { is a } \Gamma \text {-cover of } X\} .
$$

Note that if there is no such cover, then $\Gamma\left(E_{0} \hookrightarrow E_{1}\right)=\infty$. 
The clever point-set topology of Dranishnikov's paper is contained in the following proposition.

Proposition 5.1 Suppose, in the fiber pair diagram above, $B$ is path-connected and the inclusion $F_{0} \hookrightarrow F_{1}$ is nullhomotopic. Then

$$
\operatorname{cat}\left(E_{1}\right) \leq \operatorname{dim}(B)+\Gamma\left(E_{0} \hookrightarrow E_{1}\right) .
$$

The proof rests on two subtle point-set-theoretical results, which we state here - without proof - for easy reference.

Theorem 5.2 (1) [7, Theorem 1.4] If $\mathcal{U}=\left\{U_{0}, U_{1}, \ldots, U_{g}\right\}$ is an open cover of a normal space $X$, then for every $m \geq g$ there is an open cover $\mathcal{V}=$ $\left\{V_{0}, V_{1}, \ldots, V_{m}\right\}$ such that

- each $V_{k}$ is a disjoint union of open sets $W_{\alpha}$ such that $W_{\alpha}$ is contained in at least one of the sets $U_{j}$, and

- any subcollection consisting of $g+1$ sets of $\mathcal{V}$ covers $X$.

(2) [20; 7] If $\operatorname{dim}(X) \leq d$, then for any open cover $\mathcal{U}$ of $X$ and every $m \geq d+1$, there is an open cover $\mathcal{W}=\left\{W_{0}, W_{1}, \ldots, W_{m}\right\}$ such that

- $\mathcal{W}$ refines $\mathcal{U}$, and

- each $x \in X$ is contained in at least $m-d+1$ sets of $\mathcal{W}$.

Assuming Theorem 5.2, we now proceed to the proof of Proposition 5.1.

Proof of Proposition 5.1 Let $\operatorname{dim}(B)=d$ and let $\Gamma\left(E_{0} \hookrightarrow E_{1}\right)=g$.

Choose a $\Gamma$-cover of $E_{1}$ and use Theorem 5.2(1) to extend it to a cover $\mathcal{W}=$ $\left\{W_{0}, W_{1}, \ldots, W_{d+g}\right\}$ such that each $W_{i}$ can be deformed, over $B$, into $E_{0}$, and such that any subcollection of $g+1$ sets of $\mathcal{W}$ cover $E_{1}$.

Next, find a cover of $B$ by open sets which are contractible in $B$, so that the fibration $p_{1}$ is trivial over each set in the cover. Then use Theorem 5.2(2) to obtain a cover $\mathcal{V}=\left\{V_{0}, V_{1}, \ldots, V_{d+g}\right\}$ of $B$ such that each $V_{i}$ is contractible in $B$ and each point $b \in B$ is contained in at least $g+1$ of the sets $V_{i}$.

Write $\widehat{V}_{i}=p_{1}^{-1}\left(V_{i}\right)$. We claim that the collection

$$
\mathcal{C}=\left\{\widehat{V}_{0} \cap W_{0}, \widehat{V}_{1} \cap W_{1}, \ldots, \widehat{V}_{d+g} \cap W_{d+g}\right\}
$$

is an LS cover of $E_{1}$. It suffices to show that for each $b \in B, p_{1}^{-1}(b) \subseteq \cup \mathcal{C}$. If $x \in p_{1}^{-1}(b)$ then, without loss of generality, $b \in V_{0} \cap V_{1} \cap \cdots \cap V_{g}$ and $E_{1}=$ $W_{0} \cup W_{1} \cup \cdots \cup W_{g}$. Therefore - again without loss - we have $x \in W_{0}$ and hence

$$
x \in\left(p_{1}^{-1}\left(V_{0} \cap V_{1} \cap \cdots \cap V_{g}\right)\right) \cap W_{0}=\left(\widehat{V}_{0} \cap \widehat{V}_{1} \cap \cdots \cap \widehat{V}_{g}\right) \cap W_{0} \subseteq \widehat{V}_{0} \cap W_{0} .
$$

This shows that $\mathcal{C}$ covers $E_{1}$. 
It remains to show that each set $\widehat{V}_{i} \cap W_{i} \in \mathcal{C}$ is contractible in $E_{1}$. To see this, first contract each piece vertically into $E_{0}$, which can be done because the sets $W_{k}$ are $\Gamma$-sets. Then use homotopy lifting to contract each set horizontally into $F_{0}$. Finally, use the hypothesis that the inclusion $F_{0} \hookrightarrow F_{1}$ is nullhomotopic to contract to a point in $E_{1}$.

Remark 5.3 (1) It suffices to have each fiber of $p_{0}$ contractible in $E_{1}$.

(2) The conditions are satisfied if $F_{1}$ is $(r-1)$-connected and $F_{0}$ is $(r-1)-$ dimensional.

Proposition 5.1 is the bulk of Dranishnikov's [7, Lemma 3.2]. The other part involves bounding the $\Gamma$ term in the special dimension-and-connectivity case mentioned in the Remark above, but we will accomplish this in a more theoretically satisfying way below.

\section{The case of $\tilde{X}_{1} \hookrightarrow \tilde{X}$}

Let $X$ be a CW complex. Write $\pi=\pi_{1}(X)$ and let $\tilde{X} \rightarrow X$ be the universal cover. Choose a CW decomposition of $X$ and pull it up to provide $\tilde{X}$ with a $\pi$-invariant CW decomposition so that the covering map is cellular. Then we may form the fiber pair

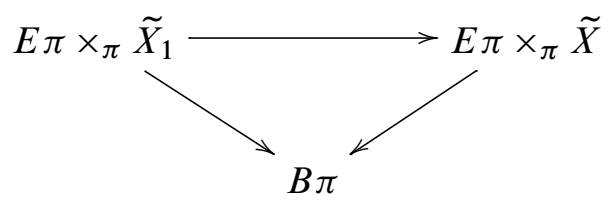

whose induced map of fibers is $\tilde{X}_{1} \hookrightarrow \tilde{X}$. It turns out that the fiber pair invariant

$$
\Gamma_{1}(X)=\Gamma\left(E \pi \times_{\pi} \tilde{X}_{1} \hookrightarrow E \pi \times_{\pi} \tilde{X}\right)
$$

is an old friend.

Theorem 6.1 $\Gamma_{1}(X)=\operatorname{cat}^{1}(X)$.

There is a fibration $q: E \pi \times_{\pi} \tilde{X} \rightarrow X$ whose fiber is the contractible space $E \pi$. It follows that $q$ is a homotopy equivalence, and so we obtain Rudyak's conjecture.

Corollary 6.2 If a connected space $X$ has fundamental group $\pi$, then

$$
\operatorname{cat}(X) \leq \operatorname{dim}(B \pi)+\operatorname{cat}^{1}(X) \leq \operatorname{dim}(B \pi)+\left\lceil\frac{\operatorname{dim}(X)-1}{2}\right\rceil .
$$

Proof This follows from Proposition 5.1, Theorem 6.1 and Proposition 4.4. 
Proof of Theorem 6.1 Let us call an open set $U \subseteq E \pi \times_{\pi} \tilde{X}$ a $\Gamma_{1}-$ set if it can be deformed over $B \pi$ into $E \pi \times_{\pi} \tilde{X}_{1}$; and let us call a set $U \subseteq X$ a cat ${ }^{1}$-set if it can be deformed in $X$ into $X_{1}$.

Suppose $U \subseteq X$ is a cat ${ }^{1}$-set; then let $\tilde{U}=p^{-1}(U) \subseteq \tilde{X}$. We show that $E \pi \times_{\pi} \tilde{U} \subseteq$ $E \pi \times_{\pi} \tilde{X}$ is a $\Gamma_{1}$-set. To see this, first form the pullback squares

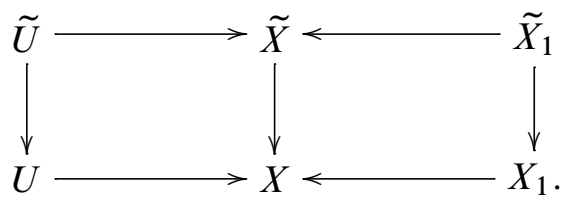

Since $U \rightarrow X$ factors through $X_{1}$ up to homotopy we may apply the homotopy lifting property to see that $\tilde{U}$ factors through $\tilde{X}_{1}$ up to $\pi$-equivariant homotopy. The claim follows because the Borel construction $E \pi \times_{\pi}(-)$ is functorial and converts equivariant homotopies to homotopies over $B \pi$.

If $X=U_{0} \cup U_{1} \cup \cdots \cup U_{n}$ is a cat ${ }^{1}$-cover of $X$, then $\tilde{X}=\tilde{U}_{0} \cup \tilde{U}_{1} \cup \cdots \cup \tilde{U}_{n}$ is a $\pi$-equivariant cover of $\tilde{X}$, and so

$$
E \pi \times_{\pi} \tilde{X}=\left(E \pi \times_{\pi} \tilde{U}_{0}\right) \cup\left(E \pi \times_{\pi} \tilde{U}_{1}\right) \cup \cdots \cup\left(E \pi \times_{\pi} \tilde{U}_{n}\right)
$$

is a $\Gamma_{1}-$ cover of $E \pi \times_{\pi} \tilde{X}$. Thus $\Gamma_{1}(X) \leq \operatorname{cat}^{1}(X)$.

Since the fibration $q: E \pi \times \pi \tilde{X} \rightarrow X$ with fiber $E \pi$ is a homotopy equivalence, it has a section $\sigma: X \rightarrow E \pi \times_{\pi} \tilde{X}$. Let $U \subseteq E \pi \times_{\pi} \tilde{X}$ be a $\Gamma_{1}$-set, and let $V=\sigma^{-1}(U)$. Then the diagram

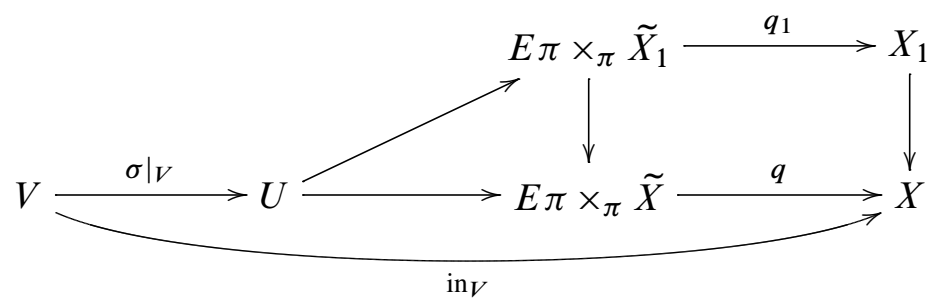

commutes on the nose, except for the triangle, which commutes up to homotopy (over $B \pi)$. This shows that $V$ is a cat ${ }^{1}$-set. Finally, if $E \pi \times_{\pi} \tilde{X}=U_{0} \cup U_{1} \cup \cdots \cup U_{n}$ is a cover of $E \pi \times_{\pi} \tilde{X}$, by $\Gamma_{1}$-sets, then we may set $V_{k}=\sigma^{-1}\left(U_{k}\right)$, and $X=$ $V_{0} \cup V_{1} \cup \cdots \cup V_{n}$ is a cat ${ }^{1}$-cover of $X$. Thus cat ${ }^{1}(X) \leq \Gamma_{1}(X)$ which completes the proof of Theorem 6.1. 


\section{Some consequences for symplectic manifolds}

A closed manifold $X^{2 n}$ is said to be cohomologically symplectic (or c-symplectic) if there is a class $\omega \in H^{2}(X ; \mathbb{R})$ such that $\omega^{n} \in H^{2 n}(X ; \mathbb{R}) \cong \mathbb{R}$ is nontrivial. Every symplectic manifold is, of course, c-symplectic. Note that the standard cuplength and dimension inequalities for LS category imply that

$$
n \leq \operatorname{cat}(X) \leq 2 n .
$$

We now compute the 1-category of c-symplectic manifolds.

Proposition 7.1 A c-symplectic manifold $\left(X^{2 n}, \omega\right)$ has cat $^{1}(X)=n=\frac{1}{2} \operatorname{dim}(X)$.

Proof By Proposition 4.4, we have

$$
n \leq \operatorname{nil}(K) \leq \operatorname{cat}^{1}(X) \leq\left\lceil\frac{\operatorname{dim}(X)-1}{1+1}\right\rceil=\left\lceil\frac{2 n-1}{2}\right\rceil=n,
$$

since $\omega \in K=\operatorname{ker}\left(H^{*}(X ; \mathbb{R}) \rightarrow H^{*}\left(X_{1} ; \mathbb{R}\right)\right)$.

Since $\pi$ free implies $\operatorname{dim}(B \pi)=1$, we obtain the following.

Corollary 7.2 If $\left(X^{2 n}, \omega\right)$ is c-symplectic and $\pi_{1}(X)$ is a free group, then $n \leq$ $\operatorname{cat}(X) \leq n+1$.

Now, by the cuplength lower bound and the (\$) upper bound, a simply connected c-symplectic manifold $\left(X^{2 n}, \omega\right)$ always has cat $(X)=n=\frac{1}{2} \operatorname{dim}(X)$. Therefore, the estimate above is saying that the effect of a free fundamental group on category is minimal. By the result of Hillman discussed in the Introduction, 4-dimensional manifolds with free fundamental group have category 2 , so the fundamental group has no effect on category in this special case.

A general c-symplectic manifold $\left(X^{2 n}, \omega\right)$ has $\operatorname{cat}(X)=n+k$ for $k \geq 0$. Then, for $\pi=\pi_{1}(X)$, we have by Corollary 6.2,

$$
\begin{aligned}
\operatorname{cat}(X) & \leq \operatorname{dim}(B \pi)+\operatorname{cat}^{1}(X) \\
n+k & \leq \operatorname{dim}(B \pi)+n \\
k & \leq \operatorname{dim}(B \pi),
\end{aligned}
$$

and here we can see how a perturbation of LS category away from its value in the simply connected case constrains the fundamental group.

The strongest instance of this occurs for the symplectically aspherical c-symplectic manifolds. A manifold $\left(X^{2 n}, \omega\right)$ is a symplectically aspherical manifold if there is a 
class $\widetilde{\omega} \in H^{2}\left(K\left(\pi_{1}(X), 1\right)\right)$ such that $f^{*}(\widetilde{\omega})=\omega$, where $f$ is the classifying map of the universal cover. This has the immediate consequence that

$$
\operatorname{dim}(B \pi) \geq 2 n=\operatorname{dim}(X) .
$$

In particular (as shown in [14]) if $\mathbb{Z}^{k}$ is the fundamental group of a symplectically aspherical (symplectic) manifold $\left(X^{2 n}, \omega\right)$, then $k \geq 2 n$. By [22], it is also true that $\operatorname{cat}(X)=2 n$, so our estimate says little about this case. However, if the estimate $(\star)$ of the Problem 1.1 holds, then we recover the stronger result $\operatorname{dim}(B \pi) \geq 2 n$ as well.

Gompf has shown [11] that every finitely presented group occurs as the fundamental group of a closed symplectic manifold of dimension 4. However, taking restricted classes of symplectic manifolds can constrain the groups that are allowed as fundamental groups. By the discussion above, we see that a free group can never be the fundamental group of a symplectically aspherical manifold.

Let's consider another restricted class of symplectic (or c-symplectic) manifolds. Recall that Bochner's theorem restricts the class of manifolds possessing a nonnegative Ricci curvature metric to those having $b_{1}(M) \leq \operatorname{dim}(M)$, with equality holding only for a flat torus. This result was improved (even for manifolds with almost nonnegative Ricci curvature) in [18] to $b_{1}(M) \leq \operatorname{cat}(M)$, again with equality only for a flat torus. Moreover, for c-symplectic manifolds, the inequality was even better.

Theorem 7.3 [18] If $\left(X^{2 n}, \omega\right)$ is a closed c-symplectic manifold with infinite fundamental group and nonnegative Ricci curvature, then

$$
b_{1}(X) \leq 2 \operatorname{cat}(X)-\operatorname{dim}(X),
$$

where $b_{1}(X)$ is the first Betti number.

This leads to an analogous estimate involving $\operatorname{dim}(B \pi)$.

Theorem 7.4 If $\left(X^{2 n}, \omega\right)$ is a closed c-symplectic manifold with infinite fundamental group $\pi$, nonnegative Ricci curvature and satisfying $(\star)$, then

$$
b_{1}(X) \leq 2 \operatorname{cat}(X)-\operatorname{dim}(X) \leq \operatorname{dim}(B \pi) .
$$

Proof If $\pi$ has infinite cohomological dimension, then there is nothing to prove, so suppose $\operatorname{dim}(B \pi)<\infty$.

$$
\begin{aligned}
\operatorname{cat}(X) & \leq\left\lceil\frac{\operatorname{dim}(B \pi)+\operatorname{dim}(X)-1}{2}\right\rceil \\
& \leq \frac{\operatorname{dim}(B \pi)}{2}+n \\
2 \operatorname{cat}(X)-2 n & \leq \operatorname{dim}(B \pi) .
\end{aligned}
$$


In fact, the inequality $b_{1}(X) \leq \operatorname{dim}(B \pi)$ follows from the Cheeger-Gromoll splitting together with the fact that groups and finite index subgroups have the same cohomological dimension. The interest in the two inequalities is that $2 \operatorname{cat}(X)-\operatorname{dim}(X)$ fits in between.

Example 7.5 Prime examples of symplectic manifolds with nonnegative Ricci curvature are products $X=T^{2 k} \times \mathbb{C P}^{n-k}$. It is easy to show that $\operatorname{cat}\left(T^{2 k} \times \mathbb{C P}^{n-k}\right)=n+k$. These products are, of course, instances of $X=B \pi \times \mathbb{C P}^{n}$, and if the estimate $(\star)$ of Problem 1.1 holds, then Theorem 7.4 is sharp since $b_{1}(X)=2 k, \operatorname{dim}(X)=2 n$, $\operatorname{cat}(X)=n+k$ and $\operatorname{dim}\left(B \mathbb{Z}^{2 k}\right)=2 k$ :

$$
2 k \leq 2(n+k)-2 n=2 k \leq 2 k .
$$

Therefore, we see that solving Problem 1.1 has interesting consequences.

\section{References}

[1] PS Alexandrov, Combinatorial topology. Vol. 1, 2 and 3, Dover Publications, Mineola, NY (1998) MR1643155

[2] C Allday, J Oprea, A c-symplectic free $S^{1}$-manifold with contractible orbits and cat $=\frac{1}{2}$ DIM, Proc. Amer. Math. Soc. 134 (2006) 599-604 MR2176029

[3] I Berstein, On the Lusternik-Schnirelmann category of Grassmannians, Math. Proc. Cambridge Philos. Soc. 79 (1976) 129-134 MR0400212

[4] M Clapp, D Puppe, Invariants of the Lusternik-Schnirelmann type and the topology of critical sets, Trans. Amer. Math. Soc. 298 (1986) 603-620 MR860382

[5] O Cornea, G Lupton, J Oprea, D Tanré, Lusternik-Schnirelmann category, Math. Surveys and Monogr. 103, Amer. Math. Soc. (2003) MR1990857

[6] A N Dranishnikov, On the Lusternik-Schnirelmann category of spaces with 2dimensional fundamental group, Proc. Amer. Math. Soc. 137 (2009) 1489-1497 MR2465675

[7] A N Dranishnikov, The Lusternik-Schnirelmann category and the fundamental group, Algebr. Geom. Topol. 10 (2010) 917-924

[8] A N Dranishnikov, MG Katz, Y B Rudyak, Small values of the LusternikSchnirelmann category for manifolds, Geom. Topol. 12 (2008) 1711-1727 MR2421138

[9] A N Dranishnikov, Y B Rudyak, On the Berstein-Svarc theorem in dimension 2, Math. Proc. Cambridge Philos. Soc. 146 (2009) 407-413 MR2475974

[10] J C Gómez-Larrañaga, F González-Acuña, Lusternik-Schnirelmann category of 3manifolds, Topology 31 (1992) 791-800 MR1191380 
[11] R E Gompf, A new construction of symplectic manifolds, Ann. of Math. (2) 142 (1995) 527-595 MR1356781

[12] D P Grossman, An estimation of the category of Lusternik-Shnirelman, C. R. (Doklady) Acad. Sci. URSS (N.S.) 54 (1946) 109-112 MR0018820

[13] J A Hillman, $\mathrm{PD}_{4}$-complexes with free fundamental group, Hiroshima Math. J. 34 (2004) 295-306 MR2120518

[14] R Ibáñez, J Kȩdra, Y Rudyak, A Tralle, On fundamental groups of symplectically aspherical manifolds, Math. Z. 248 (2004) 805-826 MR2103543

[15] A Lundell, S Weingram, The topology of CW complexes, Univ. Ser. Higher Math. VIII, Van Nostrand Reinhold, New York (1969)

[16] T Matumoto, A Katanaga, On 4-dimensional closed manifolds with free fundamental groups, Hiroshima Math. J. 25 (1995) 367-370 MR1336904

[17] R Nendorf, N Scoville, J Strom, Categorical sequences, Algebr. Geom. Topol. 6 (2006) 809-838 MR2240916

[18] J Oprea, Category bounds for nonnegative Ricci curvature manifolds with infinite fundamental group, Proc. Amer. Math. Soc. 130 (2002) 833-839 MR1866039

[19] J Oprea, Y Rudyak, Detecting elements and Lusternik-Schnirelmann category of 3manifolds, from: "Lusternik-Schnirelmann category and related topics (South Hadley, MA, 2001)”, (O Cornea, G Lupton, J Oprea, D Tanré, editors), Contemp. Math. 316, Amer. Math. Soc. (2002) 181-191 MR1962163

[20] P A Ostrand, Dimension of metric spaces and Hilbert's problem 13, Bull. Amer. Math. Soc. 71 (1965) 619-622 MR0177391

[21] F Roth, On the category of Euclidean configuration spaces and associated fibrations, from: "Groups, homotopy and configuration spaces", (N Iwase, T Kohno, R Levi, D Tamaki, J Wu, editors), Geom. Topol. Monogr. 13, Geom. Topol. Publ., Coventry (2008) 447-461 MR2508218

[22] Y B Rudyak, J Oprea, On the Lusternik-Schnirelmann category of symplectic manifolds and the Arnold conjecture, Math. Z. 230 (1999) 673-678 MR1686579

[23] H Seifert, W Threlfall, Seifert and Threlfall: a textbook of topology, Pure and Applied Math. 89, Academic Press, New York (1980) MR575168 With a preface by Joan S Birman, With "Topology of 3-dimensional fibered spaces" by Seifert

[24] B Strom, Personal communication (2008)

[25] J Strom, Lusternik-Schnirelmann category of spaces with free fundamental group, Algebr. Geom. Topol. 7 (2007) 1805-1808 MR2366179

[26] A S Švarc, The genus of a fibered space, Trudy Moskov. Mat. Obšč. 10 (1961) 217-272 MR0154284 
Department of Mathematics, Cleveland State University

Cleveland $\mathrm{OH}, 44115$

Department of Mathematics, Western Michigan University Kalamazoo MI, 49008-5200

j.oprea@csuohio.edu, jeff.strom@wmich.edu

http://academic.csuohio.edu/oprea_j/,

http://homepages.wmich.edu/ jstrom/

Received: 15 December $2009 \quad$ Revised: 22 April 2010 